\title{
Photosynthesis of the isogenic lines Triticum aestivum $\mathrm{L}$.
}

\author{
Semilet T.V. ${ }^{1 *}$, Silantyeva M.M. ${ }^{2}$, Khlestkina E.K. ${ }^{1}$ \\ ${ }^{I}$ Federal Research Center the N.I. Vavilov All-Russian Institute of Plant Genetic Resources (VIR), St. Petersburg, Russia \\ ${ }^{2}$ Altai State University, Barnaul, Russia \\ *e-mail:semilet_tatyana@mail.ru
}

Key words: photosynthesis, quantum yield $\mathrm{Y}_{(\mathrm{II})}$, electron transport rate (ETR), isogenic lines

Motivation and Aim: Currently the searching and mapping of gene loci are widely use in molecular genetics. The expression of genes influences on the operation of photosystems and other physiological processes in higher plants. The photosyntesis influences good yield of wheat. The soft wheat (Triticum aestivum L.) is an important object for these studies. Wheat is known to be occupy the largest cultivated areas among other cereals in the world. Analysis of chlorophyll fluorescence parameterswas conducted for the initial and the isogenic lines of soft wheat (i:S29Pp-Alpp-D1pp3, i:S29Pp-A1Pp-D1Pp3PF, i:S29Pp-A1Pp-D1pp3P, i:S29Pp-A1pp-D1Pp3P, i:S29Pp-Alpp-D1Pp3PF, i:S29Pp-A1Pp-D1pp3PF, i:S29pp-Alpp-D1pp3, i:S29Pp-A1Pp-DIPp3P). We established the relationship between the intensity of the photosystems and the genotype.

Methods and Algorithms: The parameters of chlorophyll fluorescence were measured by PAM- fluorimetr JUNIOR-PAM (Walz, Germany) during all stages of ontogeny. The objects of the study included the recurrent cultivar of soft wheat - Saratovskaya-29 and its isogenic lines. This isogenic lines were obtained from the donor varieties (Purple Feed and Purple). The material for the study and genetic maps was kindly provided by The Federal Research Institute of Cytology and Genetics (Novosibirsk, Russia) [2].

Results: The isogenic line-iPF2A had high and stable parameters of quantum yield and electron transport rates among other objects. The results obtained for S-iPF2A were associated with a marker Xgwm372 [1], which was inherited from the donor T. aestivum Purple (Pp3). We associated the genes expression the marker Xgwm372 with influence on a fluorescence of chlorophyll and a pigment count at different developmental stages (locus Qchc.iiwbr-2A) [1].

Conclusion: We got results of data photosyntesis for isogenic lines of soft wheat Saratovskaya-29 using method PAM-fluorimetry. The locus gene on chromosome 2A was identified based on the available published data. The transformation of this locus may changes the work of photosynthetic apparatus.

Acknowledgements: This work was conducted within the framework of the state task regarding to Theme No. 0481-2019-0001 "Genomic and postgenomic technologies for searching for new genetic markers of economically important traits and new allelic variants of agricultural valuable genes in the gene pool of cultivated plants and their wild relatives".

References

1. Bhusal N. et al. Mapping QTLs for chlorophyll content and chlorophyll fluorescence in wheat under heat stress. Biologia Plantarum. 2018;62(4):721-731.

2. Tereschenko O.Y. et al. The D Genome Carries a Gene Determining Purple Grain Colour in Wheat. Cereal Research Communications. 2012;40(3):334-341. 